\title{
Broad-band spectral evolution of GRB afterglows
}

\author{
N. Masetti ${ }^{1}$, E. Pian $^{1}$, E. Palazzi ${ }^{1}$, L. Amati ${ }^{1}$, T.J. Galama ${ }^{2}$, L. Nicastro ${ }^{3}$, P.M. Vreeswijk ${ }^{2}$, F. Frontera ${ }^{1}$, and J. van \\ Paradijs $^{2}$ \\ 1 Istituto Te.S.R.E., CNR, via Gobetti 101, I-40129 Bologna, Italy \\ 2 Astronomical Institute "Anton Pannekoek", Kruislaan 403, 1098 SJ Amsterdam, The Netherlands \\ 3 I.F.C.A.I., CNR, via Ugo La Malfa 153, I-90146 Palermo, Italy
}

Received December 29, 1998; accepted April 21, 1999

\begin{abstract}
Quasi-simultaneous Spectral Energy Distributions (SEDs) at different epochs of four among the most intensively observed Gamma-Ray Burst (GRB) afterglows have been constructed in order to investigate and compare the temporal evolution of their multiwavelength emission. The data suggest that generally the peak frequency moves toward lower frequencies with time. SED local maxima in the optical/IR range are present in some cases, possibly due to dust absorption within the host galaxy.
\end{abstract}

Key words: methods: data analysis — gamma-rays: bursts

\section{Introduction}

The evolution of the physical parameters (Sari et al. 1998) of Gamma-Ray Burst (GRB) afterglows is of crucial importance in the knowledge of these phenomena, and can be analyzed by means of the radio-to-X-ray Spectral Energy Distribution (SED) observed at various epochs. By studying multiwavelength spectral variability and in particular the change of the frequency at which the SED maximum occurs, the powering mechanism of the afterglow, as well as its hydrodynamic evolution, may be clarified. To this aim, we have studied eight GRB afterglows which have been promptly pointed by the BeppoSAX NFI and for which both an $\mathrm{X}$-ray and optical transient have been detected. Here we report only on four of these for which well sampled SEDs are available at least at two epochs.

\section{The SEDs}

We have collected the $\mathrm{X}$-ray to radio data and upper limits of the four GRB afterglows of 970228, 970508, 980329 and 980703, and we have then constructed the multiwavelength spectra at various epochs for each case (Fig. 1). When known, the host galaxy contribution was subtracted from the optical and infrared (IR) measurements, if significant with respect to the OT magnitude. Dereddening for foreground Galactic absorption was made with the law by Cardelli et al. (1989). The photometric flux calibration was taken by Fukugita et al. (1995) and by Bersanelli et al. (1991) for the optical and the IR data, respectively.

Send offprint requests to: masetti@tesre.bo.cnr.it
In the following we will discuss the four GRB afterglows separately.

GRB 970228. In order to determine the optical and IR magnitudes of the OT of this GRB we subtracted from the available measurements the following host galaxy magnitudes: $B \sim 26.5$ (extrapolated), $V=25.75, R=25.3$, $I=24.45$ (Fruchter et al. 1998), $J=24.0$ (interpolated), $H=23.2$ and $K=22.8$ (Fruchter et al. 1998); next, we dereddened the data using $A_{V}=0.75$ (Fruchter et al. 1998). Three SEDs were constructed. The first contains the X-ray data by Frontera et al. (1998a), the optical data by van Paradijs et al. (1997) and Guarnieri et al. (1997) the latter ones were corrected for the contribution of the nearby K star - and the radio upper limits by Frail et al. (1998). Note that the optical points by Guarnieri et al. (1997) were collected about 4 hours earlier than those of van Paradijs et al. (1997), that is when, according to those authors, the OT luminosity was still rising. This could explain the difference in the flux level between the two data sets. The second SED shows the ASCA (Yoshida et al. 1997) and ROSAT (Frontera et al. 1998b) X-ray data, the optical points and upper limits by van Paradijs et al. (1997), and the radio upper limits by Frail et al. (1998a). The last SED includes $V, I$ (interpolated between the HST $V$ and $I$ data sets by Fruchter et al. 1998) and $R$ (Metzger et al. 1997) optical points, $J$ and $K$ (Soifer et al. 1997) IR data, and radio upper limits by Frail et al. (1998a).

GRB 970508. Among GRB afterglows, this is the best monitored at all wavelengths, therefore five SEDs could be constructed. The first four SEDs were obtained with the data by Piro et al. (1998; X-rays), Galama et al. (1998a; $U)$, Sokolov et al. (1998; BVRI), Castro-Tirado et al. $(1998 ; R)$, Pedersen et al. $(1998 ; R)$, Morris et al. (1997; $K)$, Bremer et al. $(1998 ; \mathrm{mm})$ and Frail et al. (1997; radio). The fifth SED contains the data gathered by Galama et al. (1998b) and the 12- $\mu$ m ISO measurement by Hanlon et al. (1998). The host galaxy contribution was not removed since it was negligible at the considered epochs due to its faintness (Zharikov et al. 1998). Optical and IR data were dereddened with $A_{V}=0.08$ (Galama et al. 1998a).

GRB 980329. To obtain the first SED for this GRB afterglow we used the data by in't Zand et al. (1998; 


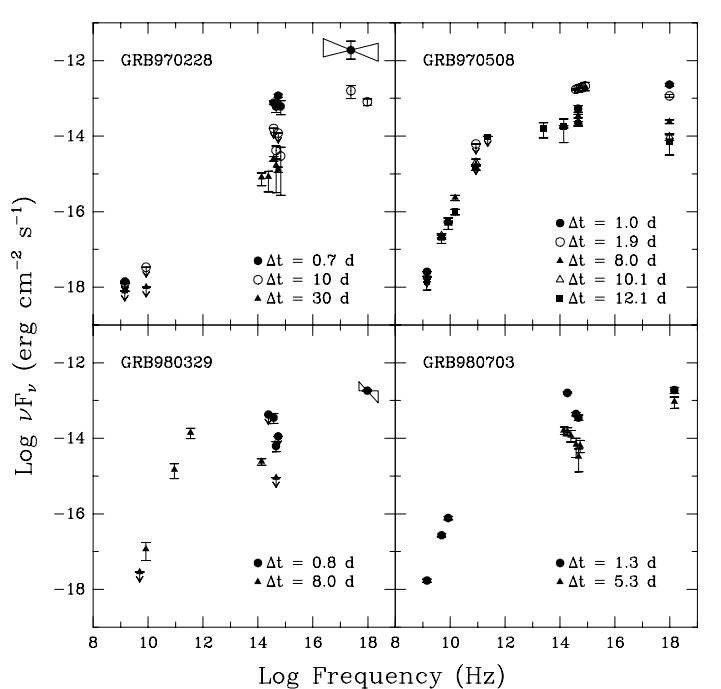

Fig. 1. SEDs of GRB afterglows at various epochs after the $\gamma$ burst. For each GRB we define $\Delta t=t_{\mathrm{obs}}-t_{0}$, where $t_{\mathrm{obs}}$ is the epoch of the observation, in days, referring to a specific SED. A larger version of this figure can be found at the URL http://tonno.tesre.bo.cnr.it/ masetti/seds.html

X-rays), Palazzi et al. (1998; $V, R$ ) and Rhoads et al. (1998a; I). The second SED was constructed with observations by Djorgovski et al. (1998; $R$ ), Metzger (1998; $K)$, Smith et al. (1998; mm) and Taylor et al. (1998, radio), made about 8 days after the burst. No host galaxy contribution has been subtracted, since its brightness is uncertain. Correction for foreground Galactic absorption $A_{V}=0.4$ (Palazzi et al. 1998) was applied to the optical and IR data. We note that the revision of the $I$ band photometric measurement on March 29.9 (Rhoads et al. 1998a) suggests a very sharp and narrow local spectral peak. If true, this would be inconsistent with a single optical-to-X-ray emission component. The change of the continuum spectral slope occurring in the optical has been tentatively explained as Lyman- $\alpha$ absorption up to $z \sim 5$ (Fruchter 1999).

GRB 980703. Two SEDs for this GRB afterglow were constructed using the X-ray data by Vreeswijk et al. (1999), the optical data by Bloom et al. (1998), Vreeswijk et al. (1999) and Rhoads et al. (1998b) and, for the first epoch, the radio data by Frail et al. (1998b). Host galaxy magnitudes $V=23.04, R=22.58, I=21.95, J=20.87$, $H=20.27$ and $K=19.62$ (Vreeswijk et al. 1999) were subtracted from the measurements. A foreground absorption $A_{V}=0.019$ (Vreeswijk et al. 1999) was taken into account. The optical/IR bump could be accounted for by intrinsic extinction in the OT environment at the source redshift ( $z=0.966$; Djorgovski et al. 1998b).

\section{Results and discussion}

From this GRB afterglow sample we find that in general, at early epochs after the GRB ( $\$ 2$ days), the SED exhibits a maximum in the $\mathrm{X}$-rays. However, due to the lack of data in the far and extreme ultraviolet range (100 to
$3500 \AA$ ), and to the large uncertainties on the X-ray spectral slope, we cannot exclude that the SED peak is located between the optical and the $\mathrm{X}$-rays. While the afterglow of GRB 970508 exhibits a smooth and continuously curved spectrum from the radio to the $\mathrm{X}$-ray frequencies, which might suggest the presence of a single emission component (presumably synchrotron radiation; e.g., Sari et al. 1998), the other cases reported in Fig. 1 show local maxima (visible on the reported SEDs as "humps") in the optical/IR range. If intrinsic, these would imply that at least two emission components build up the afterglow radio-to-Xray continuum. However, they could be the signature of gas and dust absorption within the host galaxy, which increases toward the ultraviolet frequencies. Although, due to the scarcity and irregular sampling of the data, it is extremely difficult to follow in detail the temporal evolution of the SED maximum, the data suggest that for most GRB afterglows the peak moves toward lower frequencies with time. The timescale of this displacement is hard to assess; it could be estimated for future GRBs by simultaneous monitoring in many bands, on long time intervals.

\section{References}

Bersanelli M., Bouchet P., Falomo R., 1991, A\&A 252, 854

Bloom J.S., et al., 1998c, ApJ 508, L21

Bremer M., et al., 1998, A\&A 332, L13

Cardelli J.A., Clayton G.C., Mathis J.S., 1989, ApJ 345, 245

Castro-Tirado A.J., et al., 1998, Sci 279, 1011

Djorgovski S.G., et al., 1998a, GCN 41

Djorgovski S.G., et al., 1998b, ApJ 508, L17

Frail D.A., et al., 1997, Nat 389, 261

Frail D.A., et al., 1998a, ApJ 502, L119

Frail D.A., et al., 1998b, GCN 141

Frontera F., et al., 1998a, ApJ 493, L67

Frontera F., et al., 1998b, A\&A 334, L69

Fruchter A.S., 1999, ApJ 512, L1

Fruchter A.S., et al., 1998, ApJ (in press) (astro-ph/9807295)

Fukugita M., Shimasaku K., Ichikawa T., 1995, PASP 107, 945

Galama T.J., et al., 1998a, ApJ 497, L13

Galama T.J., et al., 1998b, ApJ 500, L97

Guarnieri A., et al., 1997, A\&A 328, L13

Hanlon L., et al., 1998, A\&A (submitted)

in't Zand J.J.M., et al., 1998, ApJ 505, L119

Metzger M.R., 1998, IAU Circ. 6874

Metzger M.R., et al., 1997, IAU Circ. 6631

Morris M., et al., 1997, IAU Circ. 6666

Palazzi E., et al., 1998, A\&A 336, L95

Pedersen H., et al., 1998, ApJ 496, 311

Piro L., et al., 1998, A\&A 331, L41

Rhoads J., et al., 1998a, GCN 157

Rhoads J., Downes R., Christensen J., 1998b, GCN 144

Sari R., Piran T., Narayan R., 1998, ApJ 497, L17

Smith A.I., et al., 1998, A\&A (submitted) (astro-ph/9811026)

Soifer B., et al., 1997, IAU Circ. 6619

Sokolov V.V., et al., 1998, A\&A 334, 117

Taylor G.B., et al., 1998, ApJ 502, L115

van Paradijs J., et al., 1997, Nat 386, 686

Vreeswijk P.M., et al., 1999 (this volume)

Yoshida A., et al., 1997, IAU Circ. 6593

Zharikov S.V., Sokolov V.V., Baryshev Y.V., 1998, A\&A 337, 356 